\title{
Sulla ricezione cinquecentesca del Furioso nelle arti: traduzioni possibili?
}

La réception du Roland furieux à la Renaissance : quelles possibilités de traductions?

The Renaissance reception of Furioso into arts: possible translations?

\section{Anna Langiano}

\section{OpenEdition}

\section{Journals}

Edizione digitale

URL: http://journals.openedition.org/cei/1363

DOI: $10.4000 /$ cei. 1363

ISSN: 2260-779X

\section{Editore}

UGA Éditions/Université Grenoble Alpes

\section{Edizione cartacea}

Data di pubblicazione: 1 novembre 2013

Paginazione: $71-91$

ISBN: 978-2-84310-234-9

ISSN: 1770-9571

\section{Notizia bibliografica digitale}

Anna Langiano, «Sulla ricezione cinquecentesca del Furioso nelle arti: traduzioni possibili?», Cahiers d'études italiennes [Online], 17 | 2013, online dal 01 mai 2015, consultato il 26 mars 2021. URL: http:// journals.openedition.org/cei/1363 ; DOI: https://doi.org/10.4000/cei.1363 


\title{
SULLA RICEZIONE CINQUECENTESCA DEL FURIOSO NELLE ARTI: TRADUZIONI POSSIBILI?
}

\author{
Anna Langiano \\ Université de Rome-Tor Vergata
}

È impossibile non tradurre un'opera d'arte: ogni atto di lettura è, effettivamente, una traduzione. Inconsapevolmente assorbiamo solo alcuni degli innumerevoli spunti che ci porge la creazione artistica; selezioniamo, amplifichiamo, insomma modifichiamo:

comprendere è sempre necessariamente scambiare, comparare, trasferire, sostituire, vale a dire tradurre (che potrebbe mai voler dire comprendere quando venisse a mancare la possibilità di sostituire un segno con un altro, una parola con un'altra, una frase con un'altra, e conseguentemente venisse meno la possibilità di intendere il rimando al senso? $)^{\mathrm{I}}$

La domanda da porsi nell'affrontare lo studio della ricezione dell'Orlando Furioso nel Cinquecento è allora non se siano possibili delle traduzioni-ricreazioni del testo ariostesco, ma a che livello in questa ricreazione agisce una collettività quando un'opera viene canonizzata, quando cioè alla lettura personale si affianca e spesso sostituisce la messe di interpretazioni e rielaborazioni che ne certificano e in parte determinano l'accettazione collettiva.

"L'une des pistes prometteuses de la recherche sur l'Arioste est de se demander en quoi ont consisté les multiples stratégies qui ont conduit à la véritable canonisation $d u$ Roland furieux. $»^{2}$ Guardare un'opera con gli occhi dei suoi contemporanei ci permette di intravvedervi riflesso lo sguardo dell'autore, uomo del suo tempo, e di scrostare patine ermeneutiche che

I. C. Di Martino, Il problema della traduzione, in «Doctor virtualis», Quaderno n. 7, Milano, Cuem, 2007, p. 7I.

2. M. Ciccuto, Ce qu'il reste de l'Arioste. Les "fables " du Roland furieux et la tradition figurée, in M. Paoli e M. Preti (ed.), L'Arioste et les Arts, Paris, Éditions du Louvre / Milano, Officina libraria, 20I2, p. I44. 
il tempo e la sedimentazione culturale hanno ormai steso, velando i colori originari dell'opera.

Sono in questo senso le traduzioni meno potentemente creatrici che più fedelmente ci avvicinano - perché per questo sono state pensate - alla percezione originaria dell'opera, alla reazione comune che il testo poteva suscitare. L'originalità, la potenza creatrice sfrutta le opacità dell'opera, aumenta l'entropia, plasma con elementi conosciuti il nuovo laddove gli artigiani della cultura, gli illustratori, gli intrattenitori delle piazze concentrandosi programmaticamente sui gusti del pubblico si consacrano a un significato solo, a quello che per loro è più ovvio, più immediato; e ci indicano intuitivamente quali elementi erano maggiormente interessanti per il pubblico dell'epoca.

Nel caso del Furioso, la straordinaria varietà delle forme attraverso cui è stato accolto e diffuso il poema — forme non solo letterarie ma appartenenti ai più diversi linguaggi artistici - ci aiuta a mettere a fuoco la duttilità del capolavoro ariostesco. Si tratta di immagini del poema ovviamente forzate, semplificate e adattate a una forma irriducibile a quella concepita da Ariosto. Infatti,

[...] l'ouvre, qu'elle soit littéraire ou artistique, ce sont certes des idées, mais exprimées par une certaine forme, qui ne peut pas être remplacée par une autre forme sinon en faisant perdre à l'ouvre ce qui fait sa spécificité et donc sa valeur; c'est cette forme qui est la voie unique et obligée pour accéder au fond. ${ }^{3}$

Nel momento in cui si traspone l'Orlando Furioso, l'Orlando Furioso di Ludovico Ariosto cessa di esistere. È dunque necessario chiedersi cos'è ciò che nasce dall'opera e al posto dell'opera, e se quest'esistenza abbia una qualche autonomia o sia da considerarsi una versione di seconda mano dell'opera stessa, e quindi da espungere, o ancora se essa mantenga almeno un riverbero del poema originario e quindi abbia un senso studiarlo a fini non meramente descrittivi e conservativi.

La complessità dei legami tra le diverse forme della ricezione ariostesca permette inoltre di far risaltare i meccanismi della cultura e delle influenze, mai diretti e stagnanti ma al contrario complessi e stratificati, in un continuo dialogo tra tradizione alta e bassa e tra linguaggi artistici non univoci ${ }^{4}$.

3. M. Paoli, L'ouvre d'art hors d'elle-même?, in Ivi, p. Io.

4. Un esempio emblematico è quello riportato da Carlo Alberto Girotto: «Va anche detto, più in generale, che il mezzo dell'incisione ebbe un'incidenza non trascurabile nella fortuna figurativa del Furioso: studi recenti hanno permesso di documentare casi significativi di ripresa di queste immagini in cicli di affreschi e in oggetti 
È per questo motivo che nel seguente saggio ho scelto di rendere nel modo più ampio possibile - sebbene necessariamente non esaustivo - la varietà delle modalità di ricezione dell'Orlando Furioso nel Cinquecento. Partendo dalla complessità intellettuale dei primi commenti del poema per arrivare alla fruizione popolare basata sulla messa in musica delle ottave attraverso la resa figurativa delle illustrazioni nelle varie edizioni del poema, l'intento è mostrare come la conoscenza culturale del Furioso sia avvenuta attraverso la confluenza di forme alte e basse, afferenti ad ambiti diversi e addirittura perdendo il nesso diretto e preferenziale con il testo stesso.

Certo, nella traduzione in immagini o musica del poema, delle infinite sfumature dei versi di Ariosto viene copiata una tonalità soltanto, coll'inesorabile e conseguente appiattimento cromatico. Eppure è proprio questo il motivo dell'interesse per tali traslitterazioni mancate, per queste traduzioni immancabilmente stonate e semplicistiche del testo ariostesco: riportare alla luce nella tonalità originaria caratteristiche del poema che il tempo ha smorzato e reso irriconoscibile.

Vedremo come in più di un caso il colore che è stato recepito all'epoca non è quello che ci aspetteremmo oggi. In altri casi — casi che forse dovrebbero essere accolti con ancora maggiore stupore, se si pensa alla distanza temporale e culturale che ci allontana da Ludovico Ariosto — sì.

D'altronde

[...] ce livre, qui fut sans doute le plus grand "classique» de son siècle, put jouir [...] d'un niveau d'appréciation certainement lié au fait que ses premiers lecteurs sétaient fait de lui l'image d'un texte insaisissable, à l'instar du personnage d'Angélique, qui en est le moteur narratif - ce qui ne mettait en rien ces mêmes lecteurs en mesure de saisir l'un des principaux vecteurs de sens de ce chef-d'ouvre.

Non può quindi stupire se nel corso del Cinquecento l'Orlando Furioso ebbe una diffusione stratificata e obliqua ${ }^{6}$. Il poema, apprezzato dai più

d'uso quotidiano, quali boccali e maioliche.» (C. A. Girotto, Qualche appunto sulla ricezione dell'Orlando furioso: fortuna editoriale e figurativa, lettura e lettori, in «Chroniques italiennes web", 22, I/2012, p. 9)

5. M. Ciccuto, Ce quill reste de l'Arioste. Les " fables » du Roland furieux et la tradition figurée, cit., p. I45.

6. Per la diffusione dei poemi cavallereschi cfr. M. Roggero, Senza frontiere. Letture dei poemi cavallereschi nell'Italia moderna, in "Schifanoia», 28/29, 1995. Per la diffusione del modello ariostesco, cfr. E. Cerulli, L'Orlando furioso nella storia culturale, in Atti del Convegno Int. "L. Ariosto" (27 sett.-5 ott. 1974), Accademia Nazionale dei Lincei, Roma, 1975, pp. II-2I; M. Murrin, The Allegorical Epic. Essays in its Rise and Declin, Chicago-London, The University of Chicago Press, 1980; A. R. Ascoli, Ariosto's Bitter Harmony. Crisis and Evasion in the Renaissance, Princeton, Priceton U. P., 1987; M. Sherberg, Rinaldo. Character and intertext in Ariosto and Tasso, Saratoga, Anima Libri, 1993; D. Javitch, Ariosto classico, Milano, Mondatori, 1999; S. Jossa, La fondazione di un genere. Il poema eroico tra Ariosto e Tasso, Roma, Carocci, 20or; K. W. Hempfer, Letture discrepanti: la ricezione dell'Orlando Furioso nel Cinquecento, Modena, Panini, 2004; G. Fumagalli, La fortuna dell'Orlando Furioso in Italia nel secolo XVI, in "Atti e memorie della Deputazione ferrarese di storia patria", 
fini letterati e dal popolo analfabeta ${ }^{7}$, venne affrescato nei palazzi nobiliari, musicato dai cantastorie nelle piazze italiane, stampato in edizioni di pregio vòlte a porlo sullo stesso piano dei classici greci e latini ma anche in edizioni in ottavo, in vendita nelle fiere paesane ${ }^{8}$. La ricchezza del poema si prestò a innumerevoli letture, pronte a contraddirsi e a contaminarsi: posto al centro delle discussioni tra intellettuali sulla possibilità di un'epica moderna, diffuso tra gli strati popolari attraverso una tradizione orale giunta fino ai nostri giorni ${ }^{9}$, promotore di una vera e propria rivoluzione nell'ambito delle illustrazioni tipografiche, il poema diventa un caleidoscopio in grado di rifrangere tutta la contraddittoria complessità del Cinquecento, diventando l'«espressione più vera e piena dei [...] valori vitali ed estetici [del Rinascimento]» ${ }^{\mathrm{I}}$.

Il primo termine di paragone per comprendere come il Furioso veniva letto nel Cinquecento sono i commenti coevi, che hanno il merito di testimoniare quali caratteristiche del poema erano recepite con più interesse dal pubblico contemporaneo; non bisogna però dimenticare che queste letture riportano una visione parziale, non solo perché distanti dalla percezione che del poema avevano gli strati sociali inferiori, ma perché programmaticamente concepite come giustificazione ideologica del poema contro le immediate critiche mosse dai neoaristotelici. Le discussioni intorno all'Orlando Furioso conobbero il massimo sviluppo tra gli anni Quaranta

20, I912, pp. 133-497; G. Agnelli e G. Ravegnani, Annali delle edizioni ariostesche, Bologna, Zanichelli, 1933. L'influsso del Furioso nelle arti minori e persino negli oggetti di consumo è riscontrabile in J. Bentini (a. cura di), Signore cortese e umanissimo. Viaggio intorno a Ludovico Ariosto, Venezia, Marsilio, 1994, in particolare si vedano i saggi di L. G. Boccia, Curiosa di armamentaria ariostesca, e di C. Ravanelli Guidotti, L'Ariosto 'istoriato' sulla maiolica italiana del Cinquecento; più recentemente M. Zampetti, La fortuna dell'edizione giolitina dell'Orlando furioso nelle maioliche. Due coppe del Museo Statale di Arte Medievale e Moderna di Arezzo, leggibile in L. Bolzoni, S. Pezzini e G. Rizzarelli (ed.), "Tra mille carte vive ancora». Ricezione del Furioso tra immagini e parole, Lucca, Maria Pacini Fazzi, 20II, pp. 423-444 e T. Wilson, L'Arioste à table. Les illustrations du Roland furieux du peintre de majoliques Francesco Xanto Avelli, in M. Paoli e M. Preti (ed.), L'Arioste et les Arts, cit., pp. I7I-I83. Si veda anche il volume L'arme e gli amori. La poesia di Ariosto, Tasso e Guarini nell'arte fiorentina del Seicento. Catalogo della mostra. Firenze, Palazzo Pitti, Galleria Palatina, 21 giugno-2o ottobre 20oI, a cura di E. Fumagalli, M. Rossi e R. Spinelli, Livorno, Sillabe editore, 200 .

7. «Se voi praticate per le corti, se andate per le strade, se passeggiate per le piazze, se vi trovate ne' ridotti, se penetrate ne' musei, mai non sentite altro che o leggere o recitar l'Ariosto. Anzi, che dico corti, che dico musei? Se nelle case private, nelle ville, ne' tuguri stessi e nelle capanne ancora si trova e si canta continuamente il Furioso?» (G. Malatesta, Della nuova poesia, overo delle difese del Furioso, Verona, Delle Donne, I589, pp. I37I38), cit. in M. Roggero, op. cit., p. I22. Nel saggio già citato, Girotto rileva come Michel de Montaigne nel suo viaggio in Italia si stupisse della conoscenza del Furioso dimostrata dal popolo: cfr. C. A. Girotto, Qualche appunto sulla ricezione dell'Orlando furioso: fortuna editoriale e figurativa, lettura e lettori, cit., pp. IO-I3.

8. Cfr. E. Pace, Aspetti tipografico-editoriali di un best seller del secolo XVI: l'Orlando furioso, in «Schifanoia», III, I987, p. I09.

9. Ad esempio, come si vedrà oltre, alle narrazioni dei pupari siciliani e dei cuntastorie napoletani.

Io. W. Binni, Dai commenti del Cinquecento all'incomprensione dell'età barocca, in ID., Metodo e poesia di Ludovico ariosto e altri studi ariosteschi, a cura di R. A. Pettinelli, Firenze, La Nuova Italia, 1996, p. 334. 
e Ottanta del Cinquecento, in coincidenza con l'approfondimento dello studio della Poetica di Aristotele. Già nel 1549 Simon Fornari riportava nella sua Apologia brieve sopra tutto l'Orlando Furioso, premessa al primo esteso commento del poema, le principali critiche mosse dagli aristotelici al Furioso ${ }^{\text {II }}$ la molteplicità di azioni, di contro all'unitarietà prevista dalle norme aristoteliche, le intrusioni del narratore e la mancata corrispondenza tra titolo dell'opera e soggetto dell'opera. In risposta agli attacchi dell'ortodossia aristotelica, i difensori del Furioso puntarono a presentare il poema ariostesco come la naturale filiazione moderna dell'epoca classica, sottolineando i ritorni classici presenti nel Furioso ${ }^{12}$ : ad esempio, contro le obiezioni sulla mancata coincidenza fra titolo dell'opera e argomento rappresentato, Fornari utilizza proprio l'esempio dell'Iliade, che pur narrando l'ira di Achille non da essa prende il suo nome; fautori e detrattori di Ariosto utilizzano dunque le stesse armi per provare la grandezza o le carenze del capolavoro ariostesco.

Un altro elemento di difesa del poema ariostesco, e in particolare della moltelicità narrativa, è l'esempio classico delle Metamorfosi ovidiane; non a caso nel Cinquecento i parallelismi tra le Metamorfosi e il Furioso sono più volte discussi e in alcuni casi persino ricreati letterariamente attraverso l'opera di traduzione del testo latino. Ludovico Dolce traduce le Metamorfosi in ottava rima, creando una complessa dinamica tra i due testi: se infatti Ovidio permette di canonizzare l'Orlando contro i suoi detrattori aristotelici, a sua volta il Furioso diventa veicolo privilegiato di lettura delle Metamorfosi per il pubblico coevo.

Le Trasformationi del Dolce furono stampate dal Giolito nel 1553 in un'edizione identica dal punto di vista tipografico alle ristampe giolitine del Furioso; nella sua traduzione, Giolito aveva trasformato gli esametri ovidiani in ottave di endecasillabi e diviso i quindici libri delle Metamorfosi in trenta canti, inserendo all'inizio di ogni canto exordia modellati sugli incipit ariosteschi (sui quali erano state mosse severe critiche dagli aristotelici) e alla fine dei canti interventi del narratore rivolti ai lettori. Quel che è più interessante è però che Dolce «imitates the tantalizing interruptions of narrative that characterize canto end in the Furioso by often finishing his

II. È interessante notare come i primi attacchi all'Orlando Furioso ci siano giunti non direttamente ma attraverso le difese dei fautori dell'Ariosto; l'Arte poetica del Minturno appare nel I563, il Discorso contro l'Ariosto del Sassetti fu stampato solo nel 1575.

I2. Fondamentalmente isolata nel panorama delle discussioni cinquecentesche intorno al Furioso rimane l'originale posizione di Giraldi Cinzio, che nel suo Discorso intorno al comporre dei romanzi (I554) difende l'autonomia dei romanzi cavallereschi dai poemi classici, primo fra tutti il Furioso, come prova della loro modernità. 
cantos in the midst or at the start of one of Ovid's stories» ${ }^{13}$ : questo tipo di intervento era reso possibile da paralleli stilistici e strutturali intrinseci tra i due autori, come dimostra l'insuccesso delle traduzioni in ottave di altre opere classiche, ad esempio la traduzione dell'Eneide e dell'Odissea da parte dello stesso Dolce, mai ristampate. Un grande successo arrise invece alla traduzione delle Metamorfosi dell'Anguillara (I56I), in cui si giunge a una vera e propria contaminazione del testo di Ovidio con quello di Ariosto. L'Anguillara infatti traduce molti passi delle Metamorfosi attraverso la rivisitazione che di essi aveva fatto Ariosto nel suo poema; ad esempio, nella descrizione del salvataggio di Andromeda da parte di Perseo, invece di seguire il testo originale in cui Peseo calza sandali alati, l'Anguillara scrive che Perseo giunge volando «sul Pegaseo veloce» (Metamorfosi 4, 4II) con un'evidente interpolazione dell'episodio ariostesco del salvataggio di Angelica da parte di Ruggiero ${ }^{14}$. L'episodio di Angelica liberata da Ruggiero è una delle più ricorrenti iconografie ispirate all'Orlando Furioso ${ }^{15}$ proprio perché si riallacciava a una tradizione figurativa preesistente; un analogo parallelismo riguarda il motivo di Angelica e Medoro che incidono il loro nomi sugli alberi, che si riallaccia, come ha dimostrato W. Lee ${ }^{16}$, al tema classico degli amori di Paride e Oenone.

Uno dei più lunghi interventi dell'Anguillara riguarda il breve riferimento ad Arianna abbandonata presente nelle Metamorfosi, che nella traduzione dell'Anguillara viene dilatato in un lungo lamento modellato sul lamento ariostesco di Olimpia abbandonata da Bireno, a sua volta citazione dell'Arianna ovidiana, non in riferimento alle Metamorfosi bensì alle Heroides. L'attenzione riservata dall'Anguillara al lamento di Arianna (e delle altre donne abbandonate ritratte da Ovidio) è significativa, soprattutto se si pensa all'importanza del tòpos del lamento nella musica coeva, in primo luogo in quella madrigalistica ${ }^{17}$. La scelta dell'Anguillara di correg-

I3. D. Javitch, op. cit., p. 75 .

I4. Ivi, p. 78-79.

15. Cfr. M. Ajmar, Scene dell'Orlando Furioso nella tradizione grafica e a fresco: un problema, in "Artes", I, I993, pp. 42-59; 43.

I6. R. W. Lee, Names on trees. Ariosto into art, Princeton, Princeton U. P., 1977. Lo stesso studioso si è occupato anche dell'iconografia relativa ad Angelica e Ruggiero: W. R. Lee, Ariosto's Roger and Angelica in SixteenthCentury Art: Some Facts and Hypoteses, in "Studies in Late Medieval and Renaissance Painting in Honor of Millard Meiss", New York, 1977, I, pp. 302-319.

I7. Il tópos del lamento, come la teoria degli affetti, è una delle caratteristiche che dal madrigale passeranno al nascente dramma per musica: «[...] proprio il lamento del conte Ugolino sarà il tema di uno dei primi tentativi di canto monodico, dovuto a Vincenzo Galileo. Musicisti e musicologi nuovi, rispetto ai madrigalisti polifonici, affermano e studiano la corrispondenza tra determinati elementi musicali e determinati loro effetti sull'animo umano, anche per la suggestione classicistica dell'ethos greco, ovvero per la convinzione emozionale che il messaggio espressivo trasmette all'ascoltatore» (F. Angelini, Il teatro barocco, Roma-Bari, Laterza, I993, p. 56). Nell'elenco delle musiche tratte dall'Orlando Furioso, redatto da James Haar e Antonella Balsano, si 
gere il testo ovidiano attraverso la rilettura già offerta da Ariosto del testo di Ovidio è significativa dello stretto legame percepito nel Cinquecento fra le Metamorfosi e il Furioso, così come il tentativo compiuto dal Dolce di ampliare alcuni spunti presenti nelle Metamorfosi in vere e proprie novelle, ancora una volta sul modello del Furioso. Le due opere si trovarono d'altronde unite anche all'interno delle discussioni teoriche sul valore dei romanzi moderni, contrapposti consapevolmente all'epica antica: i difensori delle ragioni del romanzo moderno, come Giraldi Cinzio, vedevano in Ovidio un modello antico di epica anticlassica, mentre proprio sulla base della sua vicinanza ai moderni Ovidio venne più volte attaccato dai Neoaristotelici: condanne delle Metamorfosi si trovano nell'Arte Poetica del Minturno (I563), nella Poetica di Jason Denores (I588) e nel Commento del Castelvetro alla poetica aristotelica. La caratteristica comune, che permetteva ai detrattori come ai difensori di unire i due poemi, era la grande varietà narrativa e la conseguente discontinuità, che rendeva impossibile l'unità classica. Mai dato alle stampe, ma composto tra il 1575 e il 1576 , è il Discorso contro l'Ariosto di Filippo Sassetti, che muoveva forti critiche alla tecnica ariostesca dell'entrelacement; secondo Sassetti, il continuo passaggio da una storia all'altra mina l'interesse del lettore verso la materia narrata.

Nel 1542 Gabriele Giolito de’ Ferrari dà alle stampe un'edizione illustrata dell'Orlando Furioso che svolgerà un importante ruolo di mediazione tra il testo dell'Ariosto e il pubblico di lettori del Cinquecento, proponendo, attraverso l'apparato testuale e illustrativo che corredava il poema, un'interpretazione volta a rispondere alle accuse mossegli proprio in quegli anni ${ }^{18}$. L'edizione presentava quarantasei brevi allegorie

trova un'altra conferma della sensibilità tardocinquecentesca per il lamento di Olimpia: esso venne musicato da S. Rossetti ( 1567 ) in un ciclo in I7 parti; singoli brani tratti dal canto X furono inoltre messi in musica da F. Baratto (I662), M. A. Mazzone (I569), B. Serafico (I575), B. Spontoni (I558), A. Gabrieli (I575). Stesso successo riscosse l'interpretazione musicale delle ottave del XXXII, XLIV e XLV canto, dedicate al lamento di Bradamante. Cfr. J. Haar e A. Balsano, L'Ariosto in musica, in M. A. Balsano (a cura di), L'Ariosto, la musica, $i$ musicisti. Quattro studi e sette madrigali ariosteschi, Quaderni della «Rivista italiana di Musicologia», Firenze, I98I, pp. 47-88.

I8. Cfr. G. Agnelli e G. Ravegnani, Annali delle edizioni Ariostee, 2 voll., Bologna, Zanichelli, I933; G. Melzi, Bibliografia dei romanzi e poemi cavallereschi italiani, II edizione accresciuta, Milano, Tosi, I838; U. Guidi, Annali delle edizioni e delle versioni dell'Orlando furioso e d'altri lavori al poema relativi, Bologna, tipografia in via Poggiale, n. 715, 186r. Per le polemiche intorno al Furioso cfr. D. Javitch, Proclaiming a classic, cit.; K. Hempfer, Letture discrepanti. La ricezione dell'Orlando furioso nel Cinquecento, Modena, Panini editore, 2004. Sui commenti del Cinquecento cfr. W. Binni, op. cit., pp. 333-342. Per una storia delle edizioni illustrate del Furioso si rimanda a U. Bellocchi e B. Fava, L'interpretazione grafica dell'Orlando Furioso, Reggio Emilia, Banca di Cr. Popolare e Coop. Reggio Emilia, I96I; M. Cerrai, Una lettura del Furioso attraverso le immagini, in «Strumenti critici», XVI, I, 200I, pp. 99-I33. È significativo che nessuna delle edizioni curate dal poeta presenti illustrazioni, se non la silografia del ritratto di Ariosto disegnato da Tiziano apparsa nell'edizione definitiva in quarantasei canti impressa da Francesco Rosso da Valenza nel 1532. Ancora vivente il poeta erano comunque apparse 
edizioni istoriate, inizialmente da silografie provenienti da altri romanzi cavallereschi (come le due illustrazioni apparse a Milano presso Agostino da Vimercate nel 1524); nel I526 un'edizione impressa da Sisto libraro in Venezia riporta una silografia in quattro scomparti, rappresentante episodi del poema, mentre la prima edizione propriamente illustrata è quella stampata a Venezia da Nicolò d'Aristotele da Ferrara, detto Zoppino, nel I530, con silografie ad inizio di ogni canto. Cfr. in proposito F. Caneparo, Il Furioso in bianco e nero. L'edizione illustrata pubblicata da Nicolò Zoppino nel I530, in «Schifanoia», vol. 34-35, 2008, pp. I65-I72. Le edizioni successive alla giolitina del 1542 sono fortemente influenzate dalle illustrazioni di Giolito: sia l'edizione Valvassori del I548, nelle cui illustrazioni «tutto appare fuso come in un quadro» (U. Bellocchi e B. Fava, L'interpretazione grafica..., cit., p. I8), sia l'edizione veneziana di Bartolomeo Imperatore (I55I). Il vero competitore del Giolito fu Vincenzo Valgrisi, che diede alle stampe nel 1556 a Venezia una edizione in quarto del Furioso con illustrazioni a pagina intera, in cui è accentuato il dinamismo delle esili figure rappresentate; in alcuni casi nelle tavole sono rappresentate carte geografiche per indicare la simultaneità spaziale degli eventi narrati. Per l'edizione Valgrisi cfr. I. Andreoli, L'Orlando furioso «tutto ricorretto et di nove figure adornato». L'edizione Valgrisi (I556) nel contesto della storia editoriale e illustrativa del poema fra Italia e Francia nel Cinquecento, in S. Fabrizio Costa (ed.), Autour du livre ancien en Normandie. Intorno al libro antico in Normandia, Bern, Berlin, Bruxelles, Frankfurt-am-Main, New York, Oxford, Wien, Peter Lang, 20II, pp. 4I-I3I. Nel I584 per l'edizione veneziana di Francesco de' Franceschi vennero per la prima volta utilizzate incisioni in rame, delle quali eccezionalmente si conosce il nome dell'autore, Girolamo Porro, attentissimo ai particolari, «le snelle figurine, i vascelli solcanti acque leggermente increspate dalla brezza, le selve piene di luce e di ombra, i paesaggi irreali vanenti nel fondo, trattati con tecnica sottile di cesellatore» (U. Bellocchi e B. Fava, L'interpretazione grafica..., cit., p. 2I). Cfr M. Preti, «... tacendo, parla per molte lingue». Girolamo Porro illustrateur de l'Orlando furioso, in M. Paoli e M. Preti (ed.), L'Arioste et les arts, cit., pp. 199-22I. Ancora sulle singole edizioni si vedano I. Andreoli (ed.), Exercices furieux. À partir de l'édition de l'Orlando furioso De Franceschi (Venise, I584), Peter Lang, 2013, E. Falaschi, Valvassori's 553 illustrations of Orlando furioso: the development of multi-narrative technique in Venice and its links with cartography, in "Bibliofilia», LXXVII, 1975; C. Fahy, L'Orlando Furioso del I532. Profilo di una edizione, Milano, Vita e Pensiero, 1989; P. Baldan, Un Furioso illustrato in "famiglia": il figurato dossiano (presunto) del 1556, in A. Franceschetti (ed.), Letteratura Italiana e arti figurative, II, Firenze, Olschki, I988; P. Coccia, Le illustrazioni dell'Orlando furioso (Valgrisi I556) già attribuite a Dosso Dossi, in "Bibliofilia», XCIII, I99I. Tali ricerche specifiche vanno contestualizzate all'interno della tradizione dei testi a stampa illustrati, per cui cfr. Prince d'Essling, Les livres à figures vénitiens de la fin du XVe siècle et du commencement du XVI', Florence-Paris, Olschki, I908; E. Borea, Stampa figurativa e pubblico dalle origini all'affermazione nel Cinquecento, in G. Previtali (ed.), Storia dell'arte italiana, II, Torino, Einaudi, I979; P. Pallottino, Storia dell'illustrazione italiana, Bologna, Zanichelli, I988; F. Sberlati, Il testo "visualizzato". Iconologia e letteratura cavalleresca, in «Intersezioni», XV, 2, 1995, pp. 313-334; V. Branca (ed.), Boccaccio visualizzato. Narrare per parole e per immagini fra Medioevo e Rinascimento, Torino, Einaudi, I999; M. Plaisance (ed.), Le livre illustré italien au $X V I^{e}$ siècle. Texte/Image, Parigi, Kliencksieck, 1999. Per il più generale rapporto tra Ariosto e le arti visive rimando innanzitutto ai recenti volumi M. Paoli e M. Preti, L'Arioste et les arts, cit., e L. Bolzoni, S. Pezzini e G. Rizzarelli (ed.), "Tra mille carte vive ancora». La ricezione del Furioso tra immagini e parole, cit.; il catalogo della mostra Donne Cavalieri Incanti Follie. Viaggio attraverso le immagini dell Orlando Furioso, a cura di L. Bolzoni e C. A. Girotto, Lucca, Maria Pacini Fazzi, 20I3. Cfr. inoltre G. Fumagalli, L'Ariosto tra i pittori, in «Emporium», LXXVII, I933; U. Bellocchi e B. Fava, L'interpretazione grafica dell'Orlando furioso, cit.; R. W. Lee, Names on trees. Ariosto into art, cit.; G. Savarese e A. Gareffi, La letteratura delle immagini nel Cinquecento, Roma, Bulzoni, I980; E. Pace, Aspetti tipografico-editoriali di un "best seller» del secolo XVI: l'Orlando furioso, cit.; N. Harris, Bibliografia testuale o filologia dei testi a stampa: definizioni metodologiche e prospettive future: convegno di studi in onore di Conor Fahy, Udine, Forum, 1999; J. Triolo, New notes to "the Pucci service" a catalogue, in "Faenza», LXXVIII, I992, pp. 87-89; M. Ajmar, Scene dell'Orlando Furioso nella tradizione grafica e a fresco: un problema, in "Artes», I, I993, pp. 42-59; C. Gnudi, L'Ariosto e le arti figurative, in J. Bentini (a cura di), Signore cortese e umanissimo. Viaggio intorno a Ludovico Ariosto, cit.; G. Alifuoco, "La sua pittura sarà corpo de la vostra poesia". Alle origini della iconografia della Gerusalemme liberata, in Io canto l'arme e 'I cavalier sovrano. Catalogo dei manoscritti e delle edizioni tassiane (secoli XVI-XIX) nella Biblioteca Nazionale di Napoli: mostra bibliografica e iconografica (Napoli, 23 ottobre 1996-Io gennaio 1997), La Biblioteca, 1996, pp. 205-220; J. Triolo, Francesco Xanto Avelli's Pucci service (I532-I533): a catalogue, parte I e II, in "Faenza», LXXIV, I998; L'arme e gli amori. La poesia di Ariosto, Tasso e Guarini nell'arte fiorentina del Seicento, catalogo della mostra Firenze, Palazzo Pitti, Galleria Palatina, 2I giugno-2o ottobre 20oI, a cura di E. Fumagalli, M. Rossi e R. Spinelli, Livorno, Edizioni Sillabe, 200I; C. Lo Rito, L'eccellenza di Bradamante. L'episodio della rocca di Tristano nelle edizioni illustrate del Cinquecento dell'Orlando furioso, in L'uno e l'altro Ariosto in corte e nelle delizie, Atti del convegno (Ferrara, I2-I5 dicembre 2007), in «Schifanoia», 34-35, 20IO, pp. 2II-2I8; G. Savarese, Ariosto "vitru- 
di Lodovico Dolce, ognuna posta in apertura di un canto; essa conteneva inoltre la Espositione di tutti $i$ vocaboli et luoghi difficili del Dolce, la Brieve dimostratione di molte comparationi et sentenze dall'Ariosto in diversi autori imitate e la Tavola di tutte ${ }^{\mathrm{I} 9}$. È chiaro l'intento di canonizzazione implicito in tale apparato ${ }^{20}$, inteso da un lato a fornire una chiave di lettura in senso didascalico, dall'altro a trarre dal poema un vero e proprio repertorio di citazioni, immagini, vocaboli, pronto per essere utilizzato come modello ${ }^{21}$. Nella sua Brieve Dimostratione, Dolce presenta l'Orlando Furioso come il moderno corrispettivo dell'Eneide, ad essa legata da motivazioni strutturali e riprese tematiche; anche quando amplierà il suo commento introducendo riflessioni sull'influenza sul Furioso di fonti non virgiliane (prime fra tutte la Tebaide di Stazio), Dolce sceglierà sempre di privilegiare una fonte soltanto, volutamente ignorando la complessità delle citazioni ariostesche per presentare il poema come una granitica emulazione dei poemi classici. Attento alle ambiguità dei rifacimenti ariosteschi sarà invece Alberto Lavezuola, che nelle Osservationi sopra il Furioso... nelle quali si mostrano tutti i luoghi imitati dall'autore nel suo poema, edito nel 1584 nell'edizione di Francesco de' Franceschi, allarga lo spettro dei modelli danteschi non solo ad altri autori della latinità oltre a Virgilio, ma addirittura alla letteratura in volgare, da Dante a Poliziano. Ma il commento di Lavezuola, proprio per l'attenzione data alle ambivalenze del dettato ariostesco, sarà completamente superato dalla prospettiva unitaria della Brieve espositione: l'interpretazione del Dolce e l'intero apparato critico dell'edizione del Giolito diventarono infatti negli anni seguenti

viano". Il Furioso e le arti visive, in "Rassegna letteraria italiana», I, I979, pp. 28-39; E. T. Falaschi, Notes on some illustrations of Ariosto's Orlando Furioso, in "La Bibliofilia», XXV, 1973, pp. I75-I88; G. Rouchès, L'interprétation $d u$ Roland furieux et de La Jérusalem delivrée dans les arts plastiques, cit.; G. Venturi, Ludovico Ariosto : portrait d'un poète dans la littérature et dans les arts visuels, in M. Paoli e M. Preti (ed.), L'Arioste et les Arts, cit., pp. 6I-72. Estremamente utili le risorse online: C. Genovesi, A. Isolani, C. Lo Rito, D. Marotta, M. Matteoli e C. Tozzini, Topic Maps and MVD for the Representation of Interpretative Variants, in Digital Humanities 2009. Conference Abstract, University of Maryland, College Park, June 22-25, 2009 (<www.mith2.umd.edu/dho9/?page_id=99>); C. Lo Rito, Le allegorie delle edizioni Giolito e Valvassori dell'Orlando Furioso, ed. elettronica, a cura di C. Acucella, M. P. Ellero, G. Iorio, C. Lo Rito, S. Sabia e F. Tarantino, in L'Orlando furioso e la sua traduzione in immagini. Catalogo digitale, marzo 2009 (<www.ctl.sns.it/furioso/apps_v3/mastro_furioso/intro.php>).

19. Orlando furioso di M. Ludovico Ariosto novissimamente alla sua integrità ridotto et ornato di varie figure. Con alcune stanze del S. Aluigi Gonzaga in lode del medesimo. Aggiuntovi per ciascun canto alcune allegorie et nel fine una breve espositione et tavola di tutto quello che nell'opera si contiene. Con grazia e privilegio. In Venezia appresso Gabriel Iolito de' Ferrari MDXLII.

20. "All the editorial apparatus and commentary that accompanied the numerous editions of the Furioso from I540 on allegorizations, inventories of sentenze, glosses, prefaces, and commentaries advertising the poem's epic blodlines aimed either to place the poem in a prestigious tradition or to stabilize it by domesticating its errating, ambivalent meanings." (D. Javitch, Proclaiming a classic, cit., p. 69)

2I. Come vedremo più avanti, il Dolce propone un'operazione speculare nel Dialogo dei colori (I565), in cui trae dal Furioso un repertorio di esempi figurativi ad uso dei pittori contemporanei; cfr. P. Barocchi, Fortuna dell'Ariosto nella trattatistica figurativa, in EAD., Studi vasariani, Torino, Einaudi, 1984, pp. 53-67; 57-58. 
l'ineludibile termine di paragone per tutte le edizioni successive ${ }^{22}$. I rapporti tra l'Eneide e il Furioso saranno centrali nell'edizione Valgrisi del 1556, attraverso la riedizione della Vita d'Ariosto del Pigna (originariamente secondo libro de I romanzi) e le Annotationi del Ruscelli, che forniva un elenco sistematico delle filiazioni tra Eneide e Furioso "Per Carlo Re di Francia, ritrasse Latino; ... per Rodomonte, Mezentio; per Marfisa, \& Bradamante, Pantesilea, \& Camilla; per Alcina, Circe; per Cloridano, $\&$ Medoro, Niso \& Eurialo» ${ }^{23}$.

La ricerca dell'elemento moralizzante sarà seguita, fino a diventare la chiave di lettura privilegiata del poema, da Clemente Valvassori nell'edizione del Furioso del I553. In tale edizione, il rapporto del Furioso con i poemi classici passa in secondo piano, anzi Ariosto viene presentato come superiore ad Omero e Virgilio in quanto poeta della cristianità; Valvassori interpreta la mutevolezza del poema come un'allegoria della fragilità della vita e dell'inutilità delle azioni umane: "The world represented in the poem, according to his allegories, is a vale of delusion and teas where desire, carnality and blindness only lead to frustation and despair unless od's redeeming grace intervernes.» ${ }^{24} \mathrm{Nel} 1563$ presso l'editore Varisco vennero pubblicate le Allegorie di Giuseppe Horologgio; ancora nel I568 l'edizione Guerra conterrà «Nuove allegorie di Tommaso Porcacchi per ogni canto».

Al Furioso come bacino di esempi retorici si rivolge Orazio Toscanella nelle Bellezze del Furioso (I574), che mostra come le tre modalità interpretative che a partire dalla giolitina del $\mathrm{I} 542$ vengono seguite in tutte le edizioni cinquecentesche del Furioso (l'interpretazione allegorica, il riferimento ad esempi classici e la decodificazione del poema in repertori) fossero nella mentalità dei commentatori del Cinquecento profondamente connessi. Il Toscanella si propone infatti di svelare gli insegnamenti morali e le norme del buon vivere sociale offerti da Ariosto sotto il velo allegorico della narrazione epica: per comprovare i significati nascosti negli elementi narrativi dell'opera, Toscanella utilizza i poemi classici come fonti di exempla morali cui il Furioso attinge e che ripropone come un vero e

22. Secondo Agnelli-Ravegnani, la Brieve dimostratione del Dolce, oltre a essere sistematicamente ristampata nelle ventotto edizioni giolitine tra il 1542 e il 1560 , venne riedita in tredici edizioni veneziane: Comin da Trino di Monferrato, 1567; Gieromino Scotto, I567; Domenico e G. B. Guerra, 1568, 1570, 1577; Domenico Farri, I580, I594; Giovanni Alberti, I589, I597, I598; G. Domenico Imberti, I590, I6I2; Paolo Ugolino, I602.

23. Contemporaneamente al commento del Dolce era apparso il commento di Tullio Fausto da Longiano, che rilevava le corrispondenze tra il Furioso e l'Eneide, più alcuni episodi tratti dalle Metamorfosi. Nonostante l'accuratezza del commento di Fausto da Longiano, esso non venne più ristampato per oltre dieci anni, probabilmente eclissato dal successo delle edizioni giolitine contenenti il commento del Dolce, che, pur in parte dipendente dalle Annotazioni di Fausto, appariva più sistematico e di più facile consultazione.

24. D. Javitch, op. cit., p. 38. 
proprio manuale di galateo: «i duelli, i dialoghi, i doni, i colori dei vestiti dei personaggi, le imprese, ogni dettaglio insomma, diventa figura esemplare di un perfetto saper vivere in società ${ }^{25}$. Nelle edizioni successive alla giolitina i repertori tratti dal poema saranno ripresi e ampliati: tra gli esempi, la giuntina del 1544 (che contiene il Repertorio delle dichiarationi che si contengono nel libro, una Lettera di Pietro Ulivi del 3 gennaio I544, l'Epilogo delle materie de lo innamoramento di Orlando, le Citationi de' luoghi..., la Tavola di tutte le cose nel poema contenute, la Tavola brevissima delle continuationi..., la Dimostratione delle comparationi, le Discrittioni dei tempi e luochi, la Dichiaratione d'allegorie, d'historie..., gli Epitteti ed alcune elocuttioni, Breve modo di trovare nuove aggiunte...) e la prima edizione Valgrisi, del 1556 (che contiene le Annotationii, gli Avvertimenti et le Dichiarationi di Girolamo Ruscelli, la Vita dell'Ariosto del Pigna, Gli scontri de' luoghi mutati dall'Autore dopo la sua prima impressione, la Dichiaratione di tutte le favole..., il Vocabolario di tutte le parole oscure, la Tavola di tutti i nomi proprii et di tutte le materie contenute nel Furioso, la Tavola de principii di tutte le stanze del Furioso di Messer Giovan Battista Rota Padovano).

Ma l'edizione giolitina del 1542 svolge un ruolo fondamentale anche all'interno dell'affermarsi di un'iconografia del Furioso. Le illustrazioni dell'edizione perseguono infatti lo stesso disegno nobilitante del commento del Dolce, come dimostra lo studio di Marzia Cerrai ${ }^{26}$ : l'anonimo illustratore $^{27}$ ha infatti privilegiato nel ricco repertorio di storie offerto dal poema dei veri e propri cicli così da «ricondurre l'apparente molteplicità d'azione del Furioso ad una varietà di episodi, che lo stesso Aristotele, nella sua Poetica, raccomandava agli scrittori di utilizzare» ${ }^{28}$. La selezione delle scene e dei personaggi tende a dare il massimo risalto alla componente epica del Furioso, soprattutto a discapito della tematica amorosa ${ }^{29}$ (Angelica è rappresentata sempre in secondo piano), con un evidente parallelismo rispetto alle allegorie del Dolce, anch'esse tese a privilegiare avvenimenti

25. F. Magni, Dossier Ariosto: un'edizione settecentesca dell' "Orlando Furioso", tesi di dottorato discussa presso l'Università degli Studi di Roma Tor Vergata l'8 agosto 2009. Relatore: prof. Andrea Gareffi. Correlatore: prof.ssa Cristiana Lardo, p. I77.

26. Cfr. M. Cerrai, Una lettura del Furioso attraverso le immagini: l'edizione giolitiana del I542, cit., pp. 99-133. 27. Stefano Liberati e Anna Maria Voltan sostengono l'attribuzione a Jacopo Francia: S. Liberati e A. M. Voltan, Le edizioni illustrate del Furioso. Repertorio bibligorafico delle edizioni in lingua italiana dal XVI al XIX secolo, Taranto, Barbieri Selvaggi, 2007, p. 7.

28. M. Cerrai, op. cit., p. Io8.

29. Emblematica di questa svalutazione dell'elemento amoroso è la resa figurativa delle avventure di Ruggiero sull'isola di Alcina, in cui l'illustratore evita di rappresentare gli amori tra Ruggiero e Alcina per concentrarsi sulle gesta dell'eroe. 
secondari dei canti quando le azioni principali non si prestassero a un intento didascalico. Sintomatico della comunione d'intenti tra l'apparato di commento e quello figurativo è il fatto che nel 1572 le incisioni del Furioso siano state inserite dallo stesso Dolce nel suo L'Achille et l'Enea... dove egli tessendo l'historia della Iliade d'Homero a quella dell'Eneide di Virgilio, ambedue l'ha divinamente ridotte in ottava rima con argomenti et allegorie per ogni canto $^{30}$ in cui «l'ordito dei due poemi è tessuto insieme attraverso i fili del testo ariostesco, il cui modello metrico delle ottave e la cui divisione in canti, vengono applicati alla traduzione delle due opere» ${ }^{31}$.

L'importanza delle illustrazioni dell'edizione giolitina va ben oltre il consapevole tentativo di inserire il Furioso in un canone epico; esse fissano dal punto di vista dell'iconografia «un codice narrativo che si è mantenuto immutato per secoli ${ }^{32}$, basato su una tecnica multinarrativa che prevede la "compresenza di più episodi all'interno di una unità scenica»" ${ }^{33}$. Le silografie contenute nella giolitina conoscono un grande successo e vengono riprese in successive edizioni: Bartolomeo Honorati (I553), Alessandro da Viano (I565), Heredi di Pietro Deuchino (I577), Zanfretti (I582), Misserino (I596) ${ }^{34}$. Un'impostazione multinarrativa delle illustrazioni è presente anche nell'edizione del Valvassori (1553), dove «le figure intagliate in primo piano sono caratterizzate da corpi esageratamente muscolosi mentre, procedendo verso la linea dell'orizzonte, si rimpiccioliscono fino a diventare illegibili; le diverse scene sono solitamente disposte in sequenza cronologica dal primo piano al fondo con un andamento a zigzag» ${ }^{35} \mathrm{e}$ nell'edizione Valgrisi (I556), le cui illustrazioni furono attribuite a Dosso Dossi e che vantano un coerente uso della prospettiva e un piano altissimo dell'orizzonte in grado di moltiplicare il numero degli episodi narrati ${ }^{36}$. Analizzando le complesse caratteristiche di tali illustrazioni, Paolo Baldan ha rilevato «una struttura elementare di fondo tramata di rispondenze

30. Un interessante esempio è il riuso della silografia raffigurante il duello finale tra Ruggero e Rodomonte per combattimento di Enea e Turno che conclude l'Eneide; proprio tale episodio viene addotto come esempio di rilettura ariostesca dell'epica classica da Pigna (I romanzi, I554), in un continuo scambio di suggestioni fra riflessione critica e assimilazione figurativa.

3I. M. Cerrai, op. cit., p. II5. Si riscontra in questa operazione la stesso intento perseguito dal Pigna nella Vita d'Ariosto, nella quale l'autore traccia un parallelo tra la scelta di Ariosto di riprendere in mano il racconto di Boiardo e quella di Virgilio di raccontare le avventure d'Enea dopo la disruzione di Troia, dove cioè Omero si era fermato.

32. F. Zanetti, Riflessioni in margine all'interpretazione figurativa dell'Orlando Furioso, cit., p. I2.

33. Ivi, p. I2I.

34. Ivi, p. I3I, nota I9.

35. Ivi, p. I3I.

36. Per le complesse caratteristiche delle illustrazioni di quest'edizione cfr. P. Baldan, Un Furioso illustrato «in famiglian: il figurato dossiano (presunto) del I556, cit. 
analogiche» ${ }^{37}$ : il ritorno degli stessi personaggi all'interno della stessa illustrazione, resa possibile dal divario temporale interno ai canti, crea un complesso sistema di analogie e variazioni che traduce in termini figurativi l'instabilità dei personaggi e degli eventi del poema, che ci vengono presentati in atteggiamenti speculari e in continua metamorfosi. Le caratteristiche del poema vengono insomma riproposte attraverso le tecniche proprie dell'arte visiva:

[...] la successione temporale viene risolta attraverso un susseguirsi di piani prospettici senza che emergano soluzioni di continuità. Essi creano una profondità spaziale mediante un impercettibile, progressivo e dinamico passaggio dal primo piano, punto di avvio narrativo, fino allo sfondo formicolante, nel campo lungo, del momento di approdo conclusivo. ${ }^{38}$

A fine secolo la tecnica multinarrativa è ripresa dalle illustrazioni presenti nell'edizione di Francesco De' Franceschi (1584), che presenta rilevanti caratteri di novità: innanzitutto per la prima volta si tratta di illustrazioni attribuibili con certezza a un artista, Girolamo Porro; inoltre, l'illustratore dedica un'inedita attenzione agli episodi secondari del poema, ad esempio alle storie raccontate dai personaggi del poema.

È rilevante che uno dei principali cicli pittorici del Cinquecento tratti dall'Orlando Furioso ${ }^{39}$, la decorazione di Nicolò dell'Abate già in palazzo

\section{Ivi, p. 583 .}

38. Ivi, p. 582 .

39. Nel suo studio L'interprétation du Roland furieux et de La Jérusalem delivrée dans les arts plastiques, in "Études italiennes", $\mathrm{n}^{\circ}$ 3, I920, pp. I23-I4O, e $\mathrm{n}^{\circ}$ 4, pp. 193-2I2, G. Rouchès annota la mancanza di soggetti ariosteschi nella pittura del Cinquecento. Cesare Gnudi segnala, oltre ai cicli abateschi, il dipinto, attribuito a Girolamo da Carpi, Ruggero libera Angelica e conservato a El Paso, una versione tardocinquecentesca dello stesso soggetto si trovava invece a Roma, nella Collezione di Lionello Venturi (cfr. C. Gnudi, L'Ariosto e le arti figurative, in J. Bentini [a cura di], Signore cortese e umanissimo, cit., pp. 13-47). R. W. Lee ha concentrato invece le sue ricerche sulla raffigurazione di Angelica e Medoro, riallacciandola alla tradizione figurativa dell'episodio di Paride e Oenone raccontato da Ovidio e che nel Cinquecento aveva avuto una fortuna autonoma. Cfr. R. W. Lee, Names on trees. Ariosto into art, cit., 1977. Le opere segnalate dal Lee sono tutte della fine del Cinquecento, a comprovare una ricezione tardiva. Sull'impostazione «ariostesca» del ciclo ispirato all' Eneide affrescato da Nicolò dell'Abate a Scandiano, cfr. F. Sberlati, Iconologia tardogotica e letteratura cavalleresca, in J. Bentini (a cura di), Signore cortese e umanissimo, cit., pp. 75-95; 76. Per quanto riguarda le pitture parietali, oltre agli affreschi di palazzo Torfanini ora conservati a Bologna si segnalano: Parma, Palazzo del giardino; Modena, torre di Boggiovara; Bergamo, piazza Mascheroni; a Gorlago, villa Gozzini; Teglio, Palazzo Besta; Chiusa di Pesio, presso Cuneo, Osteria della Ferrata e presso la famiglia Burgo a Verzuolo; infine Frascati, Villa Mondragone. È già stato notato da Marta Ajmar (Scene dall'Orlando Furioso nella tradizione grafica e a fresco: un problema, in «Artes», I, I993, pp. 42-59) l'assoluta preponderanza del Nord Italia nella collocazione geografica delle pitture, in primo luogo ovviamente i centri d'interesse estense, con l'unica eccezione di Villa Mondragone a Frascati. A questi si devono aggiungere i cicli perduti delle rocche estensi di Sassuolo e Scandiano, per cui cfr. G. Paolozzi Strozzi, Vicissitudini storiche degli affreschi abateschi della rocca dei Boiardo a Scandiano e D. Cuoghi, Per la definizione dell'originale collocazione del Camerino dipinto e del Paradiso nella rocca di Scandiano, entrambi in J. Bentini (a cura di), Signore cortese e umanissimo, cit. Si veda in proposito soprattutto F. Caneparo, De l'art du livre à l'art de la fresque. Sur les pas de l'Arioste à travers les Alpes, in M. Paoli e M. Preti (ed.), L'Arioste et les Arts, cit., pp. I56-170. 
Torfanini a Bologna, utilizzi anch'esso tale tecnica «raccogliendo intorno a quelli principali in primo piano altri momenti della storia nell'unica visione che la finta arcata e le eleganti figure poste agli imbocchi dell'arco incorniciano sulle pareti, come sulla pagina di un libro illustrato» ${ }^{4}$. Seguendo il modello illusionistico della Camera degli Sposi di Mantegna, Nicolò dell'Abate utilizza per le pareti della camera una visione continua delle avventure di Ruggiero sull' isola di Alcina ${ }^{41}$. "Figures in action retreat behind the pillars and columns of the loggie; those in the foreground are at all times at eye level with the spectator, and their actions, read as occurring in the present, determine the temporal sequence of the painted narrative.» ${ }^{42}$ Per la compresenza di più episodi nella stessa unità prospettica, dell'Abate poteva riferirsi al modello dell'edizione giolitina del 1542 , e in alcuni punti l'artista giunge a tradire il testo ariostesco per rimanere fedele alla resa figurativa giolitina, come per la descrizione di Eurifilla ${ }^{43}$. Il più diretto rapporto con lo spazio dello spettatore della decorazione parietale rispetto alla miniatura permette a Nicolò dell'Abate di utilizzare alterazioni dello spazio per rendere figurativamente il ritmo continuamente mutevole del poema: in una delle pareti del Gabinetto, l'ordine degli episodi è disposto in maniera tale che "the image of Ruggiero changes from brave knight fighting, to courtly knight, voluptuary courtier, back to armed and fighting knight. When the foreground images are scanned from left to right, the painter seems to have depicted a different, and slapstick, scenario" ${ }^{44}$. Ancora più interessante è la scelta abatesca di modificare l'orientamento della lettura a seconda delle pareti, così che «the spatio-temporal conventions of the narrative scheme would have varied from wall to wall, another devic perhaps to reproduce pictorially the abrupt furnished by the poem» ${ }^{45}$.

In ambito pittorico ${ }^{46}$ la tradizione multinarrativa, risalente al Medioevo, giungeva fino al Quattrocento, sia nella prassi figurativa che nella trattatistica (ad esempio nel De Pictura dell'Alberti): essa persiste nonostante

40. C. Gnudi, op. cit., p. 40.

4I. Proprio questo episodio del poema rappresenta, secondo Simon Fornari nella sua Spositione sopra l'Orlando Furioso, uno dei più perfetti esempi dell'allegorizzazione ariostesca.

42. E. Langmuir, Le audaci imprese... Nicolò dell'Abate’' frescoes from Orlando furioso, in «Storia dell'arte», XLII, 198I, p. 143. Cfr. anche G. Guandalini e G. Martinelli Braglia, La torre di Baggiovara: un ciclo ariostesco di seguaci di Nicolò dell'Abate, in "Atti e Memorie della Deputazione di Storia patria per le antiche province modenesi», s. XI, vol. XI, 1989, pp. I39-I56.

43. Ivi, p. I48. Per un attento paragone delle illustrazioni giolitine e degli affreschi bolognesi cfr M. Ajamar, op. cit., p. 46.

44. Ivi, p. I49.

45. Ivi, p. I50.

46. Sugli scambi e parallelismi tra la tradizione iconografica delle illustrazioni e quella pittorica rimando a F. Sberlati, Iconologia tardogotica e letteratura cavalleresca, cit., che ricostruisce gli influssi della pittura tardogotica sulle illustrazioni dei romanzi cortesi prima e cavallereschi poi. Sberlati nota inoltre come la tradizione 
la condanna del classicismo agli inizi del Cinquecento perché permetteva di mantenere un'impostazione narrativa del racconto visivo. Nel caso dei cicli parietali ispirati al Furioso, però, la compresenza di più episodi in un'unica prospettiva si riallaccia esplicitamente all'influsso delle illustrazioni a stampa, come dimostra Marta Ajmar, nello studio già citato.

Le illustrazioni del Furioso rappresentano non solo la simultaneità temporale dei diversi episodi, ma raffigurano insieme i diversi luoghi ed elementi: «cielo, terra, acqua sono gremiti di figure, che vengono ripetute, dal primo piano al fondo, seguendo la tecnica 'a ripresa' dell'Ariosto» ${ }^{47}$.

Gli illustratori sottolineano dunque proprio l'aspetto maggiormente messo in discussione dagli avversari del poema, ovvero la sua mancanza di unità; come per i commenti in difesa dell'Ariosto, in risposta alle polemiche sulla mancanza di unità dell'Orlando Furioso e in sintonia con le nuove tendenze della cultura manierista, le illustrazioni vengono man mano affiancate da spiegazioni allegoriche. Secondo Gennaro Savarese, sono proprio i punti del Furioso maggiormente "figurativi» a prestarsi a letture allegoriche, tanto da poter essere accostabili ad artisti quali il Mantegna, Piero di Cosimo, Lorenzo Costa ${ }^{48}$; proprio nella ricerca di simili punti «qualche spinta verso la traccia buona può venire perfino dalle interpretazioni cinquecentesche del Furioso in chiave allegorizzante, prime fa tutte le varie 'sposizioni' di Simon Fòrnari» ${ }^{49}$. Il rapporto tra illustrazioni e commenti del Furioso è ambivalente: da un lato le illustrazioni concorrono insieme all'apparato critico a fornire un'interpretazione classicizzante del poema, dall'altro esse esasperano proprio l'aspetto maggiormente stigmatizzato dai contemporanei, ovvero la molteplicità narrativa. La complessità dei rapporti tra riflessione letteraria sul Furioso e sua iconografia si mostra chiaramente in queste riflessioni di Massimiliano Mussini:

Se la teoria letteraria aveva definito la forma della tragedia, restaurando un classicismo che sostanzialmente si accordava alle contemporanee espressioni dell'arte figurativa, l'interpretazione del poema ariostesco compiuta da Nicolò dell'Abate e dagli

iconografica dei libri cavallereschi non conosce quel rapido processo di standardizzazione cui sono sottoposti i libri sacri o di diritto, ciò che si traduce in una pluralità di proposte interpretative del testo istoriato.

47. F. Zanetti, Note per una storia delle illustrazioni dell'Orlando Furioso, in AA.VV., Ludovico Ariosto: documenti, immagini, fortuna critica, Presidenza del Consiglio dei Ministri, Dipartimento per l'informazione e l'editoria, Roma, 1992, pp. I29-136; I33. L'autrice ricollega alcune caratteristiche delle illustrazioni cinquecentesche alla logica della consequenzialità del Furioso, per cui cfr. G. Barlusconi, L'Orlando Furioso poema dello spazio, in AA.VV., Studi sull'Ariosto, Milano, Pubblicazioni della Università Cattolica del Sacro Cuore, 1973, pp. 39-130.

48. G. Savarese, Ariosto "vitruviano»: il Furioso e le arti visive, in "Rassegna letteraria italiana», I, I979, pp. 28-39; 55. Poi in volume in ID., Il Furioso e la cultura del Rinascimento, Roma, Bulzoni, 1984, pp. 53-70.

49. Ivi, p. 54 . 
illustratori delle successive edizioni dell' Orlando furioso seguiva esattamente il percorso inverso. Come la pittura dei primi manieristi rinunciava all'armonia classica degli immediati predecessori, così l'interpretazione figurativa del poema ariostesco abbandonava i principi dell'Umanesimo per privilegiare la funzione narrativa ed edonistica dell'immagine, in una singolare convergenza con le idee sulla poesia formulate da Castelvetro. ${ }^{50}$

Il motivo di tale complessità è da ricercarsi nell'intreccio di interpretazioni visive, commenti letterari e trattati di pittura, ognuno pronto a rileggere tematiche ariostesche secondo le proprie necessità: Ludovico Dolce nel Trattato della pittura propone Alcina quale modello di bellezza perfetta ${ }^{\text {51 }}$, degna dei colori di Tiziano, e nel successivo Dialogo sui colori (Venezia, I565) utilizza le stanze dell'Ariosto come "codice interpretativo dei colori, delle imprese, dei moti, degli affetti» ${ }^{52}$; e similmente Ariosto viene ripreso dal Rinaldi (Il monstruosissimo mostro di Giovanni de' Rinaldi, Venezia, I592) e dal Lomazzo (Trattato dell'arte della pittura, scoltura et architettura, Milano, I584) ${ }^{53}$.

Il legame del Furioso con l'interpretazione pittorica degli affetti è significativa, perché ci apre un parallelo verso la «teoria degli affetti» che nel Cinquecento fu alla base della complessa questione del rapporto tra testo e musica, nella prassi madrigalistica prima e nella nascita del melodramma poi. Nel corso del secolo si afferma infatti

[...] il carattere non imitativo bensì espressivo della parola nel madrigale monodico, in cui conta soprattutto la corretta espressione degli accenti (cioè la durata: l'intonazione e l'intensità delle note sulle sillabe toniche della parola). [...] La parola monodica è $[. .$.$] pateticamente significante, cioè privilegiata come voce umana, di scarsa$ sonorità fisica ma di intensa espressività psicologica. ${ }^{54}$

Come scriveva Gaetano Cesari, il madrigale musicale, nella forma assunta nella prima metà del Cinquecento, «non lascia scorgere alcun nesso evolutivo coll'omonima forma lirica fiorita in Firenze più di un secolo innanzi» ${ }^{5}$. Fondamentale rimane però sempre il rapporto del madrigale

50. M. Mussini, Riflessioni in margine all'interpretazione figurativa dell'Orlando furioso, in AA.VV., Ludovico Ariosto: documenti..., cit., pp. II9-I28; I26.

5I. Per la rappresentazione della bellezza della donna in poesia si rimanda a G. Pozzi, Il ritratto della donna nella poesia d'inizio Cinquecento e la pittura di Giorgione, in Letteratura italiana e arti figurative, a cura di A. Franceschetti, Firenze, Olschki, pp. 585-595.

52. P. Barocchi, Fortuna dell'Ariosto nella trattatistica figurativa, cit., p. 58.

53. Ivi, pp. 58-6o.

54. F. Angelini, Il dramma per musica, cit., p. 65.

55. G. Cesari, Le origini del madrigale cinquecentesco, Bologna, Forni, 1978, p. I. 
musicale con la parola letteraria ${ }^{56}$. Inizialmente basato sul predominio della voce soprana in grado di preservare l'«atteggiamento retorico, cioè la puntualità di una declamazione attenta agli accenti metrici del testo» ${ }^{57}$ e quindi principalmente su una «recitazione declamatoria del testo» ${ }^{58}$, il madrigale ${ }^{59}$ cinquecentesco tra il terzo e il quarto decennio del secolo opera una svolta in senso polifonico. D. T. Mace ha ricondotto questo cambiamento all'influsso della poetica del Bembo, tesa a ignorare "gli schemi puramente 'formali' di rima e metro — ed esaltare la funzione 'espressiva' di ritmo e sonorità» ${ }^{6}$. Secondo Mace, "per eguagliare i sottili effetti ritmici dei petrarchisti, i compositori avevano bisogno della polifonia» ${ }^{6}$ : solo attraverso la polifonia era possibile per il madrigale suggerire relazioni tra parole e ritmi che traducessero musicalmente «le pause tra le parole, l'indugio su certe sillabe, l'affrettarsi su altre, il variare gli schemi d'accento e quantità» ${ }^{62}$. Per questo motivo, il madrigale musicale cinquecentesco è caratterizzato da una "grande libertà e spezzatura del congegno metrico» ${ }^{63}$. La ricerca di affinità espressive tra parole e musica nella seconda metà del 'soo ha un riscontro nelle opere dei trattatisti che studiarono le analogie strutturali tra musica e parola, nella convinzione "dell'universale temperamento e consonanza armonica del creato, specchio dell'armonia celeste che poeta e musico mediano per gli uomini» ${ }^{64}$.

56. Per il rapporto tra madrigale musicale e letterario: C. Don Harràn, Tipologie metriche e musicali del madrigale ai suoi esordi, in P. Fabbri (a cura di), Il madrigale tra Cinque e Seicento, Bologna, Il Mulino, I988, pp. 95-I22. Per le caratteristiche del madrigale letterario cfr. P. G. Beltrami, La metrica italiana, Bologna, Il Mulino, 2002, pp. 287-329; G. Bertone, Breve dizionario della metrica, Torino, Einaudi, I999, p. II7; G. L. Beccaria, Dizionario di linguistica e di filologia, metrica, retorica, Torino, Einaudi, 1994; G. Gorni, Le forme primarie del testo poetico, in Letteratura italiana, III, Le forme del testo. Teoria e poesia, Torino, Einaudi, I994, pp. 487-493.

57. J. Haar, Ripercorrendo gli esordi del madrigale, in M. A. Balsano (a cura di), L'Ariosto, la musica, i musicisti, cit., p. 59 .

58. Ivi, p. 69.

59. Sul madrigale cinque-seicentesco si rimanda a L. Bianconi, Il Seicento, Storia della musica, vol. V, Torino, EDT, I991, pp. 3-32; N. Pirrotta, Introduzione al madrigale: dalle lezioni di Storia della Musica tenute nell'Università di Roma, Roma, La Goliardica, 1972, pp. 3-93. È interessante anche rilevare la geografia dei centri propulsori: da Venezia (Verdelot e Willaert), Firenze (Layolle, Arcadelt, Corteccia) e Roma (Constantio Festa e Roberto Naich), la nuova lirica si propagò a Mantova (Jachet Berchem), Ferrara (Jhan e Alfonso dalla Viola), Orvieto (forse Gero).

6o. D. T. Mace, Pietro Bembo e le origini letterarie del madrigale italiano, in M. A. Balsano (a cura di), L'Ariosto, la musica, i musicisti, cit., p. 74.

6I. Ivi, p. 82.

62. Ivi, p. 8I.

63. J. Haar, Ripercorrendo gli esordi del madrigale, cit., p. 4.

64. F. Angelini, op. cit., p. 57. 
Il primo gruppo di madrigali su testi dell'Orlando Furioso ${ }^{65}$ risale agli anni '30; singole stanze furono musicate da Arcadelt, Verdelot, Nolletto e Alfonso della Viola. A partire dagli anni '40 Francesco Bifetto, il Martoretta e Hoste da Reggio musicarono interi cicli tratti dall'opera di Ariosto. Jacquet Berchem giunse a comporre un ciclo di più di 90 stanze dall' Orlando Furioso, dedicato ad Alfonso I di Ferrara. Anche Salvatore di Cataldo e Salvatore Ricciardo composero dei cicli ispirati al poema: Tutti i principii di canti posti in musica (1559) e I madrigali del reverendo don Francesco Ricciardo, maestro di capella nella città di Cassano, sopra li principii dell'Ariosto (1600).

Lo studio della ricezione dell' Orlando Furioso nei testi musicali coevi permette di avere una comprensione, seppure indiretta, di un elemento altrimenti impossibile da rilevare, ovvero l'oralità, che all'epoca di Ariosto non era solo una modalità di fruizione ma una vera e propria ri-creazione collettiva dell'opera. È significativo che il primo riferimento che abbiamo della formazione del poema sia legato a una situazione di oralità, quale la lettura di passi del poema da parte dell'autore stesso a Isabella d'Este. L'idea di un poema composto «recitando", con quel che di cadenza c'è nell'atto di leggere ad alta voce, suscita interessanti problematiche nella sua resa orale: sappiamo che Ariosto modificava le proprie stanze dopo averle sentite cantare nelle piazze, e che Tromboncino compose un madrigale tratto dal poema sulla base del testo che circolava vocalmente, perché il madrigale, stampato nel 1517 , ha delle significative differenze rispetto al testo a stampa, ciò che prova che il madrigale testimonia una fase in cui il poema girava ancora solo vocalmente a corte ${ }^{66}$. Inoltre alcuni dei più famosi cantastorie del Cinquecento, come Jacopo Coppa di Modena o Ippolito Ferrarese, includevano brani romanzeschi dell'Ariosto nel loro repertorio ${ }^{67}$. A facilitare l'accoglienza del poema nella produzione musicale sia colta che popolare era l'uso delle ottave da parte dei cantori di tutta Italia già nei secoli precedenti la composizione del Furioso ${ }^{68}$.

Estremamente significativo della vivace sopravvivenza della tradizione orale delle ottave ariostesche è l'esperimento condotto dall'Ensemble Daedalus di Roberto Festa nell'incisione de La favola di Orlando di

65. Per un elenco delle musiche sulle ottave del Furioso si confronti A. Einstein, Orlando Furioso and La Gerusalemme liberata as Set to Music during the I6th and I7th Centuries, in "Notes», VIII, 1950/51, pp. 623630 e M. A. Balsano e J. Haar, L'Ariosto in musica, cit., pp. 50-88.

66. J. Haar, Arie per cantar stanze ariostesche, in M. A. Balsano (a cura di), L'Ariosto in musica, cit., p. 33.

67. Ivi, p. 34.

68. Cfr. l'introduzione di Roberto Festa all'incisione de La favola d'Orlando di Jachet Berchem, Accent, 20II, p. 4 del libretto. 
Jacquet Berchem (Accent Records, 20II), che accosta le ottave musicate nel Cinquecento da Jacquet Berchem a una narrazione in prosa modellata sui racconti dei pupari "to establish a thread between the old culture of the Renaissance madrigal and the living tradition of the cuntastorie and puppetteers of Sicily» ${ }^{69}$.

\section{Bibliografia}

Ajmar Marta, Scene dell'Orlando Furioso nella tradizione grafica e a fresco: un problema, in "Artes», I, I993, pp. 42-59.

Baldan Paolo, Un Furioso illustrato in "famiglia": il figurato dossiano (presunto) del I556, in A. Franceschetti (ed.), Letteratura Italiana e arti figurative, II, Firenze, Olschki, 1988.

Balsano Maria Antonella (ed.), L’Ariosto, la musica, i musicisti. Quattro studi e sette madrigali ariosteschi, Quaderni della "Rivista italiana di Musicologia", Firenze, 198I, pp. 47-88.

Barlusconi Giovanna, L'Orlando Furioso poema dello spazio, in AA.VV., Studi sull'Ariosto, Milano, Pubblicazioni della Università Cattolica del Sacro Cuore, I973, pp. 39-I30.

Barocchi Paola, Fortuna dell'Ariosto nella trattatistica figurativa, in EAD., Studi vasariani, Torino, Einaudi, I984, pp. 53-67.

Bellocchi Ugo e Fava Bruno, Linterpretazione grafica dell'Orlando Furioso, Reggio Emilia, Banca di Cr. Popolare e Coop. Reggio Emilia, I96I.

Bolzoni Lina et alii (ed.), "Tra mille carte vive ancora". Ricezione del Furioso tra immagini e parole, Lucca, Maria Pacini Fazzi, 20II, pp. 423444.

Caneparo Federica, De l'art du livre à l'art de la fresque. Sur les pas de l'Arioste à travers les Alpes, in M. Paoli e M. Preti (ed.), L'Arioste et les Arts, Paris, Éditions du Louvre / Milano, Officina libraria, 2012, pp. $156-$ I70.

-, Il Furioso in bianco e nero. L'edizione illustrata pubblicata da Nicolò Zoppino nel 1530, in «Schifanoia», vol. 34-35, 2008, pp. I65-172.

Cerrai Marzia, Una lettura del Furioso attraverso le immagini, in "Strumenti critici», XVI, I, 200I, pp. 99-I33. 
Cerulli Enrico, L'Orlando furioso nella storia culturale, in Atti del Convegno Int. "L. Ariosto" (27 sett.-5 ott. 1974), Roma, Accademia Nazionale dei Lincei, 1975, pp. II-2I.

Cesari Gaetano, Le origini del madrigale cinquecentesco, Bologna, Forni, I978.

Ciccuto Marcello, Ce qu'il reste de l'Arioste. Les "fables» du Roland furieux et la tradition figurée, in M. Paoli e M. Preti (ed.), L'Arioste et les Arts, Paris, Éditions du Louvre / Milano, Officina libraria, 2012.

Di Martino Carmine, Il problema della traduzione, in "Doctor virtualis», Quaderno n. 7, Milano, Cuem, 2007.

Fabbri Paolo (a cura di), Il madrigale tra Cinque e Seicento, Bologna, Il Mulino, 1988.

Falaschi Enid T., Notes on some illustrations of Ariosto's Orlando Furioso, in «La Bibliofilia», XXV, 1973, pp. I75-I88.

Fumagalli Elena et alii (ed.), L'arme e gli amori. La poesia di Ariosto, Tasso e Guarini nell'arte fiorentina del Seicento, Livorno, Sillabe Editore, 2006.

Genovesi Chiara, Fumagalli Elide et alii, Topic Maps and MVD for the Representation of Interpretative Variants, in Digital Humanities 2009. Conference Abstract, University of Maryland, College Park, June 22-25, 2009 (<www.mith2.umd.edu/dhog/?page_id=99>).

Liberati Stefano e Voltan Anna Maria, Le edizioni illustrate del Furioso. Repertorio bibligorafico delle edizioni in lingua italiana dal XVI al XIX secolo, Taranto, Barbieri Selvaggi, 2007.

Lo Rito Claudia, Le allegorie delle edizioni Giolito e Valvassori dell'Orlando Furioso, ed. elettronica, a cura di C. Acucella, M. P. Ellero, G. Iorio, C. Lo Rito, S. Sabia, F. Tarantino, in L'Orlando furioso e la sua traduzione in immagini. Catalogo digitale, marzo 2009 (<www.ctl.sns. it/furioso/apps_v3/mastro_furioso/intro.php>).

Girotto Carlo Alberto, Qualche appunto sulla ricezione dell'Orlando furioso: fortuna editoriale e figurativa, lettura e lettori, in "Chroniques italiennes web» 22, I/20I2.

Gnudi Cesare, L'Ariosto e le arti figurative, in Jadranka Bentini (a cura di), Signore cortese e umanissimo. Viaggio intorno a Ludovico Ariosto, Venezia, Marsilio, 1994.

Lee Rensselaer Wright, Names on trees. Ariosto into art, Princeton, Princeton U. P., 1977.

Preti Monica, «... tacendo, parla per molte lingue». Girolamo Porro illustrateur de l'Orlando furioso, in M. Paoli e M. Preti (ed.), L'Arioste et les Arts, Paris, Éditions du Louvre / Milano, Officina libraria, 20I2, pp. 199-22I. 
Roggero Marina, Senza frontiere. Letture dei poemi cavallereschi nell'Italia moderna, in "Schifanoia», 28/29, 1995.

SAVARESE Gennaro, Ariosto "vitruviano». Il Furioso e le arti visive, in "Rassegna letteraria italiana», I, I979, pp. 28-39.

Sberlati Francesco, Il testo "visualizzato». Iconologia e letteratura cavalleresca, in "Intersezioni», XV, 2, I995, pp. 313-334.

Rouchès Gabriel, L'interprétation du Roland furieux et de La Jérusalem delivrée dans les arts plastiques, Paris, E. Leroux, 1920.

Venturi Gianni, Ludovico Ariosto: portrait d'un poète dans la littérature et dans les arts visuels, in M. Paoli e M. Preti (ed.), L'Arioste et les Arts, Paris, Éditions du Louvre / Milano, Officina libraria, 20I2, pp. 6I-72.

Zanetti Francesca, Note per una storia delle illustrazioni dell'Orlando Furioso, in AA.VV., Ludovico Ariosto: documenti, immagini, fortuna critica, Roma, Presidenza del Consiglio dei Ministri, Dipartimento per l'informazione e l'editoria, I992 pp. I29-I3. 